\title{
Interactive comment on "Excitation of chorus with small wave normal angles due to BPA mechanism into density ducts" by Peter A. Bespalov and Olga N. Savina
}

\section{Anonymous Referee \#1}

Received and published: 21 June 2019

Referee report on the paper, "Excitation of chorus with small wave normal angles due to BPA mechanism in density ducts" by P. A. Bespalov and D. N. Savina

I have read the above paper with interests, and I have come to the conclusion that this paper can be acceptable for publication in Angeo, but only after the authors will make the revisions listed below.

General remark: The idea of wave-particle interactions in a confined area such as whistler ducts is very acceptable, and this is theoretically investigated in this paper. Fundamentally it can be acceptable for publication, but only after the authors will make the appropriate revisions. 
Specific remarks: (1) English The English of this paper is not good enough to be accepted in an international journal like Angeo. I strongly request the authors to polish their English with the help of an native English speaker. This will definitely enhance the quality of the paper. (2) Title: "into density ducts" is not good, and it is better to use "in density ducts". (3) Abstract, line 4 beam pulse amplifier (BPA) mechanism (4) Introduction, line 14 Something is wrong, and I can suggest the following change. -somewhat lower than and just above half the minimum - - in question (see a review by Sazhin and Hayakawa (1992)).

Sazhin, S., and M. Hayakawa, Magnetospheric chorus emissions: A review, Planet. Space Sci., vol.40, 681-697, 1992.

- Line 20; I can suggest one more paper, which is published in a not so popular journal. (Karpman and Kaufman, 1984; Ishikawa et al., 1990; Laird, 1992; _-) if you are interested in.

Ishikawa, K., K. Hattori, and M. Hayakawa, A study of ray focusing of whistler-mode waves in the outer magnetosphere, Trans. of the IEICE (Institute of Electronics, Information and Communication Engineers of Japan), Vol. E73, 149-154, 1990. You will see it as an attachment. (5) Line 16: in Bell et al. (2009). (6) Line 20iijŽ̌Must be misspelling. Laird, 1992 (7) I am very unhappy with the first paragraph of p.2. Because you have cited only the recent papers on direction finding of chorus emissions, and it seems that you are not aware of earlier work before 1990. Previous papers should be properly described in the paper.

Page 2, line 4: (Muto et al., 1987; Hayakawa et al., 1990; Santolik et al., 2009)

Muto, H., M. Hayakawa, M. Parrot, and F. Lefeuvre, Direction finding of half- gyrofrequency VLF emissions in the off-equatorial region of the magnetosphere and their generation and propagation, J. Geophys. Res., 92, 7538-7550, 1987. Hayakawa, M., S. Shimakura, M. Parrot, F. Lefeuvre, and K. Hattori, Direction finding of chorus emissions in the outer magnetosphere and their generation and propagation, Planet. Space 
Sci., 38, 135-143, 1990.

(8) The authors mention that the direction finding by Taubenschuss et al. (2014) is ANGEOD based on the assumption of a single wave. However, the earlier DF works by Muto et al. and Hayakawa et al. are much more general, because they used the wave distribution function. So, how about including the following sentences on line 9 (after the sentence of in a cold homogeneous plasma). We here compare the THEMIS results with earlier analyses based on a more general concept of wave distribution function. For the lower band chorus, the earlier work by Hayakawa et al. (1990) is very consistent with the THEMIS result. While, there is some discrepancy between Muto et al. (1987) 's result and THEMIS result for the upper band chorus. (9) Line 17: - beam pulse amplifier (BPA) mechanism of - - BPA concept appears firstly here in this paper, and you had better mention something about this BPA here. (10) Page 3, line 6: The depleted duct (e.g. Helliwell, 1965) (11) Line 7: enhanced duct (Helliwell, 1965; Karpman - (12) Line 18: well-known form (Laird, 1992) (13) Line 21: Gendrin velocity (14) Page 4, line 4: electron cyclotron (15) Page 6, line 4: recall the formation process of chorus frequency-time spectrogram in the implementation of the BPA mechanism (16) Line 9: classify the duct solutions (17) Page 7, line 7: Actually the number of -- (18) Line 14: realization of the BPA mechanism (19) Line 26: the BPA mechanism (20) References: -Addional papers should cited here in References. -p8, line 27; should be Gurnett -Heliwell (1995) seems to be not cited in the text.

Please also note the supplement to this comment: https://www.ann-geophys-discuss.net/angeo-2019-83/angeo-2019-83-RC1supplement.pdf

Interactive comment on Ann. Geophys. Discuss., https://doi.org/10.5194/angeo-2019-83, 2019.

Interactive

comment 
ANGEOD

PAPER

A Study of Ray Focussing of Whistler-Mode Waves in the Outer Magnetosphere

Interactive

comment

Kiyohiko ISHIKAWA', Nonmember, Katsumi HATTORI ${ }^{\dagger}$ and

Masashi HAYAKAWA', Members

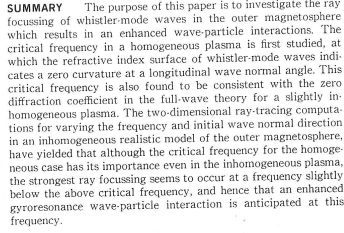

1. Introduction

Gyroresonance wave-particle interaction is impor.
tant in studying not only the generation of VLFF/ELF tant in studying not only the generation of
emissions in the magnetosphere, but also the structure emissions in the magnetosphese, enic energetic particles
and stability of the magnetospher (e. . . . Helliwell and Crystal ${ }^{[14)}$. In order to enhance
efficiently the phase-bunching of incoming gyroresonant efficently the phase-bunching of incoming gyos, the focussing
electrons by counter-propagating waves

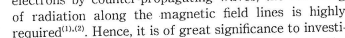
required
gate the propagation of whistler-mode waves near the gate the propagation of whele equator in a wide frequency range with normalized
nontospers frequency from 0.1 to 0.7 in the outer magnetospere,
with respect to aying a particular attention to the with respect to paying a. particular attention to
efficient wave-particle interactions. There is ample experimental and theoretical evidence of wave refrac. tion in the plasmasphere, but little study is done on the topic of wave refraction in the outer magnetospheri In Sect ? we discuss the propagation of whistler-mode waves in a homogeneous magnetospheric plasma based
on the study of refractive index surface and then a full ous, realistic magnetosphere by means of the two-dimensional ray Manuscript received July 26, 1989 . Institute of Atmos. The authors are with Research Institute of Atmos
pherics, Nagoya University, Toyokawa-shi, 442 Japan.

\section{racing computations. \\ Propagation in a Homogeneous Magnetosphere}

The propagation of whistler-mode waves in a homoindex surfaces. For dense plasmas such that the electron plasma frequency $f_{s}$ is much larger than the electron gyrofrequency $f$ the whistler mode refractive index sur. ace above and below the critical frequency of $f_{f} / 2$. Figure 1 illustrates this situation, and Fig. 1 (a) refers to the frequency below the critical frequency and fig. igure, the surface below the critical frequency is convex at small wave normal angles, while above the critical

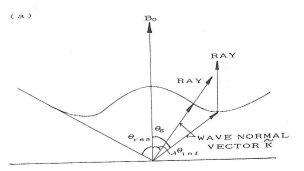

$\cdots$

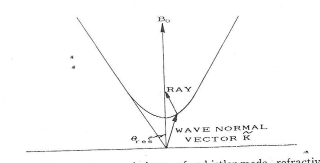

ig. 1 Change in morphology of whistler-mode refractive index surface (a lelationship of ray and wave normal
trequency. The directions is indicated. Three characteristic angles, and

Fig. 1. 